\title{
Experimental Realization of Tunable Transmission Lines Based on Single Layer SIWs Loaded by Embedded SRRs
}

\author{
Farhad Farzami, Majid Norooziarab, Student Member, IEEE
}

\begin{abstract}
Wave propagation in single layer substrate integrated waveguides (SIWs) loaded by embedded split ring resonators (ESRRs) is discussed theoretically and experimentally. It is shown that the loaded SIW can support a backward-wave passband below the cutoff frequency $\left(f_{c}\right)$ of the host SIW or a stopband will appear above $f_{c}$ if the effective transversal permeability is negative. In addition, varactor-loaded ESSRs (VLESRRs) can show resonance frequency agility through a variable reverse bias voltage applied to the varactors. Next, VLESRRs are loaded in SIWs to realize transmission lines (TLs) with either tunable passbands (stopbands) below (above) $f_{c}$ of the host SIWs. Both cases are considered in this paper. First, an SIW with $f_{c}=7 \mathrm{GHz}$ is loaded by five VLESRRs showing a tunable passband below the cutoff from 1.5 to $4 \mathrm{GHz}$. Second, an SIW with $f_{\mathrm{c}}=1.9 \mathrm{GHz}$ and five VLESRRs is discussed where a tunable stopband is achieved above $f_{c}$ from 2 to $4 \mathrm{GHz}$. In both cases, the reverse bias voltage applied to the varactors varies from 0 to $20 \mathrm{~V}$. Finally, two fabricated prototypes are provided for each case to validate the analysis. The measured and simulated results are in good agreement.
\end{abstract}

Index Terms - Backward-wave passband, narrow stopband or rejection band (notch), Substrate Integrated Waveguide (SIW), tunable Transmission Line (TL), Varactor Loaded Embedded Split Ring Resonator (VLESRR) and Wave propagation.

\section{INTRODUCTION}

A RECTANGULAR waveguide (RWG) is a common guiding structure that transmits microwave signals and has the advantages of high power handling capability and high quality factor, [1]. The structure is an inevitable part of microwave and millimeter wave systems, though the 3dimensional form has limited its application where integration with planar circuits is required. In this regard, planar transmission lines (TLs), such as microstrip, which are compact, inexpensive and can be easily integrated with microwave circuits, have surpassed waveguides. However, they suffer from a high degree of losses in higher operating frequencies.

To make use of the advantages of these two TLs, Substrate Integrated Waveguides (SIWs) were introduced. These structures have found growing interest since they preserve most of the advantages of RWGs and can also be used in planar circuits. They consist of two rows of metallic vias modeling the side walls of an RWG. Moreover, most of waveguide-based devices are extensively implemented by SIWs, such as slot antennas [2], circulators [3] and filters [4].

In recent years, there has been an attractive issue which discusses a metamaterial-based waveguide, [5]. Specifically, several resonant elements, such as Split Ring Resonators (SRRs), are etched in a slab and the slab is then loaded in the center of a conventional RWG. In this case, the SRRs effectively modify the transversal permeability to negative values in limited frequency bands. In [6], it was shown that such an SRR-loaded RWG shows two surprising results: (1) a backward- wave passband below the cutoff frequency $\left(f_{c}\right)$ of the waveguide and (2) a stopband above $f_{c}$. The first case is interpreted as a miniaturization method for RWGs while the second one corresponds to a narrow rejection band (notch) within the highpass response of the RWG.

On the other hand, to realize a planar replica for the aforementioned SRR-loaded RWG, Embedded SRRs (ESRRs) were proposed as an alternative magnetic metamaterial resonator in SIWs, $[8,9]$. These resonators, which function with their effective permeability, were designed to be compatible with the SIW topology; since SIWs are integrated in a dielectric substrate and the slab containing SRRs cannot be used in SIWs.

In addition, electric resonators such as Complementary SRRs (CSRRs) can be etched on the top or bottom ground planes of SIWs where a proper electric excitation is provided for the resonator. These resonators mainly function with their effective permittivity. The CSRR-loaded SIW is commonly used in many bandpass and bandstop filters [4], diplexers [7] and etc. Nevertheless, in this paper we consider ESRRs (frequency characteristics, wave mechanism and etc) and improve ESRR-loaded SIWs.

In all previous works, multi-layer designs were required to 
realize an ESRR-based SIW. A three layer SIW loaded by double ESRRs is completely discussed in [10]. It was shown that a passband (stopband) below (above) $f_{c}$ will appear in the frequency interval where the transversal permeability is negative.

In this paper and for the first time, wave propagation in single-layer ESRR-based SIWs is investigated theoretically and experimentally. This new single-layer configuration still allows further manipulation; i.e. loading the ESRRs by varactor diodes is possible.

Toward this end, Varactor- Loaded ESRRs (VLESRRs) are used to provide a magnetic resonator with a continuous tunable resonant frequency. Consequently, two different cases of tunable negative effective permeability below and above $f_{c}$ of a host SIW can be considered. It is shown that two TLs are achieved; one with a tunable passband below $f_{c}$ and the other one with a tunable narrow stopband (notch) above $f_{c}$. Both cases realize tunable TL modulus with full electronic control, fast sweep and easy calibration of the bands, easy fabrication process, low cost and profile.

Finally, the two prototypes of the VLESRR-based SIW TLs are fabricated and tested. The measured results are in good agreement with those obtained by simulation and analysis.

\section{SIW AND ESRR STRUCTURES}

\section{A. SIW Structure}

An SIW structure can be considered as an RWG with the side walls replaced by two rows of metallic vias. Similarly, the dominant mode is similar to the $\mathrm{TE}_{10}$ mode of RWGs with a cutoff frequency, $f_{c}$, of, [11]

$$
f_{c}^{T E_{10}}=\frac{c}{2 w_{\text {equ }} \sqrt{\varepsilon_{r}}}
$$

Where $c$ is the speed of light in vacuum, $\varepsilon_{r}$ is the relative permittivity of the substrate, $w_{\text {equ }}$ is the width of the equivalent RWG,

$$
w_{\text {equ }}=w_{S I W}-\frac{d^{2}}{0.95 \times p}
$$

In the above equation, $w_{S I W}$ is the width of SIW, $d$ is diameter of the vias and $p$ is the center-to-center spacing between them. A microstrip-to- SIW transition is also designed for the best possible matching, Fig. 1.

In addition, to operate the SIW as a waveguide, leakage losses must be small, which will make some restrictions on $d$ and $p$. To this aim, a useful design curve is provided in [12] from which the SIW structure of Fig. 1 is designed.

\section{B. Embedded SRR Structure}

Magnetic metamaterials such as SRRs can have negative effective permeability if excited properly. These resonators create an interesting phenomenon when loaded in an RWG. In a similar study, embedded SRRs (ESRRs) were used in an

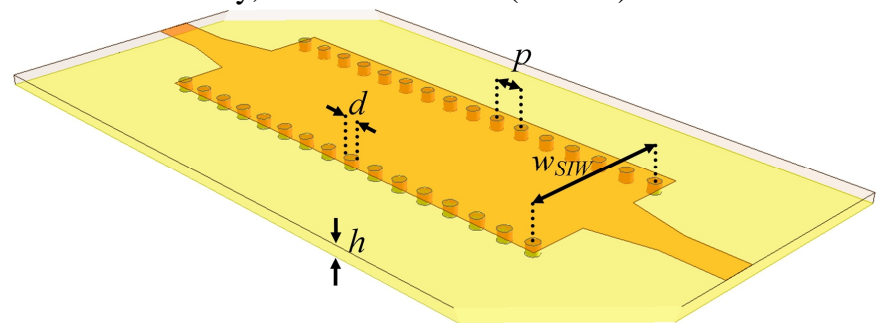

Fig. 1. Topology of a typical SIW structure.

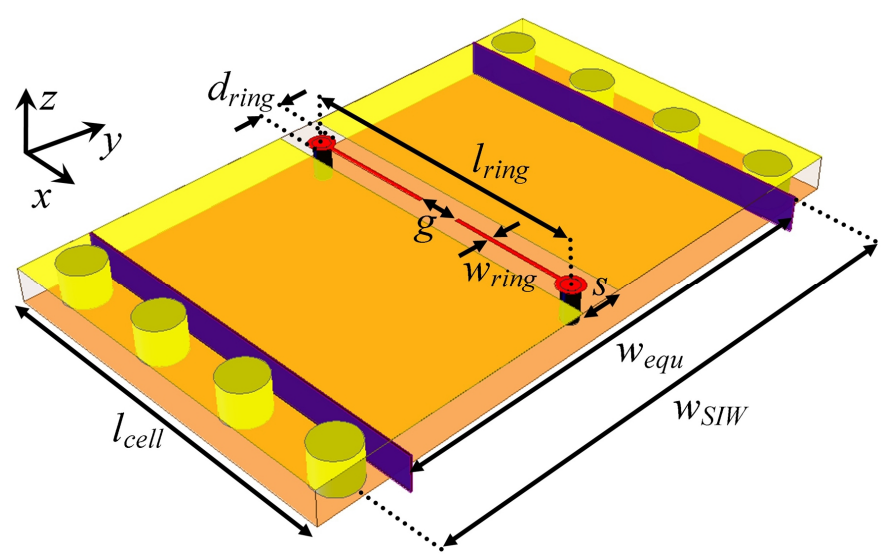

Fig. 2. A unit cell of the proposed single layer ESRR- loaded SIW.

SIW to achieve the same results as those of the SRR- loaded RWG, [10]. In this case, the ESRRs were embedded in the middle layer of a three- layer SIW. The use of three layers was inevitable to avoid the ESRRs from being short circuited. In a next study [13], an ESRR was proposed that eliminated one of the sections, namely the strip between the vias of the ESRR were replaced by a common ground plane; resulting in a two-layer structure.

A single layer ESRR-loaded SIW unit cell is shown in Fig. 2. The proposed ESRR consists of two vias connected through a common metallic plane similar to the ESSRs of [13]. The gap-coupled strip is also realized in a slit cut from the other metallic plane, Fig. 2. Hence, the need for the second section is eliminated and the ESRR can be implemented in a single layer. It should be noticed that the slit is cut from the center of the SIW plane and does not influence wave propagation of the dominate mode, since the corresponding surface currents on the plane are not perturbed, [1].

\section{Wave Mechanism \& Parameter Extraction}

The dominant $\mathrm{TE}_{10}$ mode can be represented by two plane waves propagating in a zig-zag fashion along the guide, [1]. These plane waves bounce back and forth between the side walls since an SIW with a width of $w_{\text {SIW }}$ can be modeled by an equivalent RWG with a width of $w_{\text {equ }}$. For simplicity, one of these plane waves is shown in Fig. 3(a).

For each plane wave, the Poynting vector can be considered as the superposition of a longitudinal and a transversal component. The longitudinal (transversal) component of the Poynting vector, $P_{l}\left(P_{t}\right)$, is attributed to the transversal 
(longitudinal) component of the magnetic field, $H_{t}\left(H_{l}\right)$, and the electric field, $E$, Fig. 3 (b) and (c), respectively.

As can be seen from Fig. 3(b), the transversal component of the magnetic field, $H_{t}$, is only perpendicular to the ESRR's surface which will induce currents in the ring. The induced current will then generate equivalent magnetic dipole moments. The mechanism of magnetic resonators like SRRs in presence of magnetic field is shown in [14]. The ESRR can be considered as a capacitively loaded antenna with the gap acting as the capacitance. If this antenna operates slightly above the resonant frequency, the local scattered magnetic field will be almost out of phase with the incident field. Thus, the resultant local magnetic field will be lower than the incident field. This leads to a negative magnetic polarization and consequently modifies the effective permeability in this direction. In [15] it is shown that the effective permeability of this metamaterial has the form given by

$\mu_{e f f}(f)=1-\frac{f_{m p}^{2}-f_{\circ}^{2}}{f^{2}-f_{\circ}^{2}-j \gamma f}$

Here, $f$ is the frequency of signal, $f_{m p}$ is the magnetic plasma frequency (in the lossless case, $\left.\mu_{e f f}\left(f_{m p}\right)=0\right), f_{0}$ is the resonant frequency of the ESRR, and $\gamma$ represents losses.

Now, the permeability constant will be considered for the dominate $\mathrm{TE}_{10}$ mode and in the three directions. In the longitudinal $x$ direction: the component of the magnetic field is parallel to the ESRR. Thus, the relative permeability is constant and approximately equal to that of the vacuum, $\left(\mu_{x x}\right.$ $=\mu_{\text {longitude }} \approx 1$ ). In the $\mathrm{z}$ direction: $\mu_{z z} \approx 1$ since the magnetic field has no component in this direction. In the y direction: $H_{y}$ is perpendicular to the ESRR's surface and can modify the permeability. Based on the above explanation and Fig. 3, $\mu_{x x}$ $=\mu_{z z} \approx 1$ and $\mu_{y y}$ may be negative. Therefore, the permeability will be uniaxial. The characteristic of the medium can be represented with a permeability tensor. The tensor follows the coordinate of Fig. 3,

$\bar{\mu}=\mu_{0} \bar{\mu}_{r}=\mu_{0}\left[\begin{array}{ccc}\mu_{x x} & 0 & 0 \\ 0 & \mu_{y y} & 0 \\ 0 & 0 & \mu_{z z}\end{array}\right] \approx \mu_{0}\left[\begin{array}{ccc}1 & 0 & 0 \\ 0 & \mu_{\text {eff }} & 0 \\ 0 & 0 & 1\end{array}\right]$

The above tensor demonstrates that the permeability constants in the $x$ direction (where the longitudinal component of the magnetic field is parallel to the ESRR) and in the $z$-direction (where there is no magnetic field component) remain unchanged and equal to that of vacuum. However, the permeability constant in the transversal $y$ direction is affected by the transversal magnetic field component and may be negative.

To investigate the effect of ESSR on the SIW, an approximate method can be used. In this method, the ESRR is first analyzed solely to extract the associated constitutive parameters; then the ESRR-loaded SIW unit cell is considered as an equivalent SIW filled of a homogenous medium with effective constitutive parameters extracted from

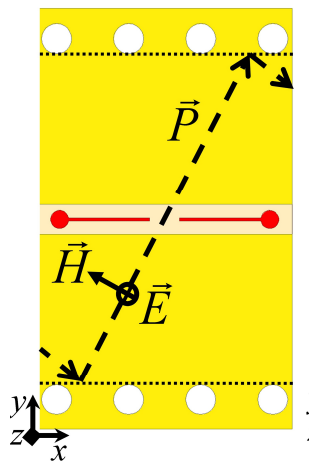

(a)

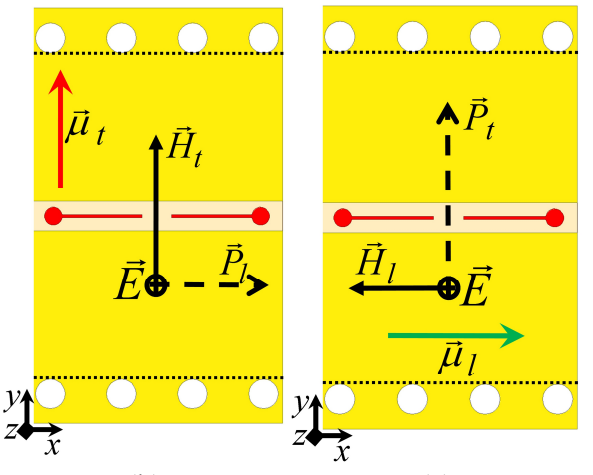

(b) (c)
Fig. 3. Wave propagation in an SIW loaded by the proposed embedded split-ring resonator (ESRR). (a) The equivalent plane wave. (b) Propagation in the transversal $y$ direction. (c) Propagation in the longitudinal $x$ direction.

the ESRR. This is the method adopted in previous studies and is already proved to be useful, $[6,10]$.

To this end, we first begin by extracting the constitutive parameters. In order to extract the constitutive parameters of an unknown material or structure, there is a simple procedure; namely, a TEM wave is incident to a unit cell of the structure and then from the transmitted $\left(S_{21}\right)$ and reflected coefficients $\left(S_{11}\right)$, the index of refraction $(n)$ and the surface impedance $(z)$ can be achieved, [16].

$$
\begin{aligned}
& z= \pm \sqrt{\frac{\left(1+S_{11}\right)^{2}-S_{21}^{2}}{\left(1-S_{11}\right)^{2}-S_{21}^{2}}} \\
& n=\frac{1}{k_{0} l_{c}} \cos ^{-1}\left\{\frac{1}{2 S_{21}}\left(1-S_{11}^{2}+S_{21}^{2}\right)\right\}
\end{aligned}
$$

In (6), $l_{c}$ is the length of the unit cell and $k_{0}$ is the free space wave number. Once the surface impedance $(z)$ and the refractive index $(n)$ are extracted from S-parameters, the constitutive parameters can be obtained from

$\varepsilon=n / z$

$\mu=n \times z$

To apply the above procedure, the unit cell of the ESRR along with the necessary boundary condition should be specified. To support TEM wave propagation and a magnetic field aligned perpendicular to the axis of the ESRR, the boundary condition is defined as that in Fig. 4. In this case, perfect electric conductors (PEC) are applied to the top and bottom walls ( $x y$ plane), and perfect magnetic conductors (PMC) are applied to the side walls ( $x z$ plane). The width of the ESRR unit cell $(\Delta y)$ is determined so that the propagated TEM wave can efficiently excite the ESRR. Moreover, the unit cell height $(\Delta z)$ is chosen to be a little greater than the thickness of the host dielectric $(h)$ to avoid short circuiting (due to PEC wall); though the distance $(\Delta z-h<\lambda / 40)$ is small 
enough to assure that the whole TEM wave passes through the unit cell.

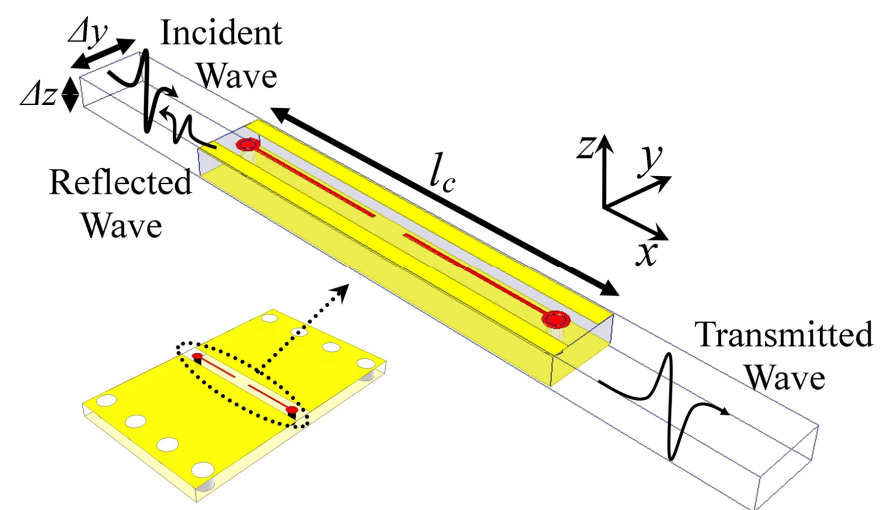

Fig. 4. Unit cell of the ESRR under periodic boundary condition with $\Delta y=2$ $\mathrm{mm}, \Delta z=0.9 \mathrm{~mm}$ and $l_{c}=8.5 \mathrm{~mm}$.

The magnitude and phase of the $\mathrm{S}$ parameters of the ESRR unit cell are sketched in Fig. 5 (a). The resultant refractive index (n) and the surface impedance, obtained from (5) and (6), are also shown in Fig. 5 (b). Finally, Eq. (7) and (8) are used to extract the complex effective permittivity and permeability. As expected, the transversal permeability has a resonance behavior and becomes negative after $6.45 \mathrm{GHz}$. In addition, the permittivity is also influenced as a consequence of the electric field which is tangential to the vias of the ESRR and can make an electric moment. However, it is important to note that the effective permittivity remains positive in the whole frequency band of interest.
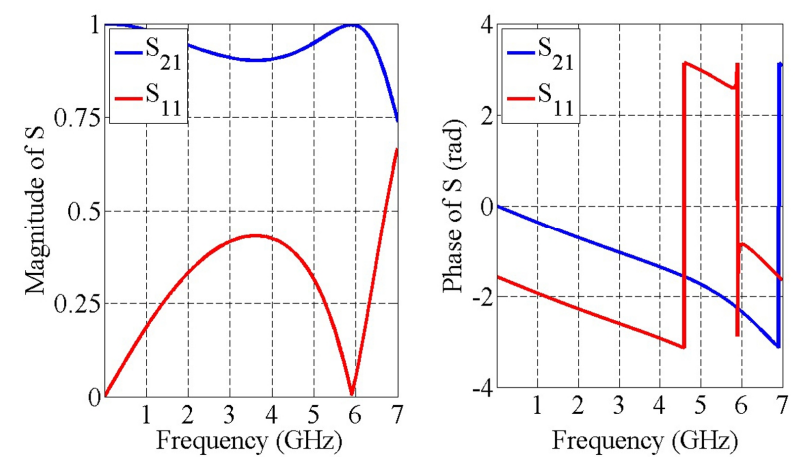

(a)
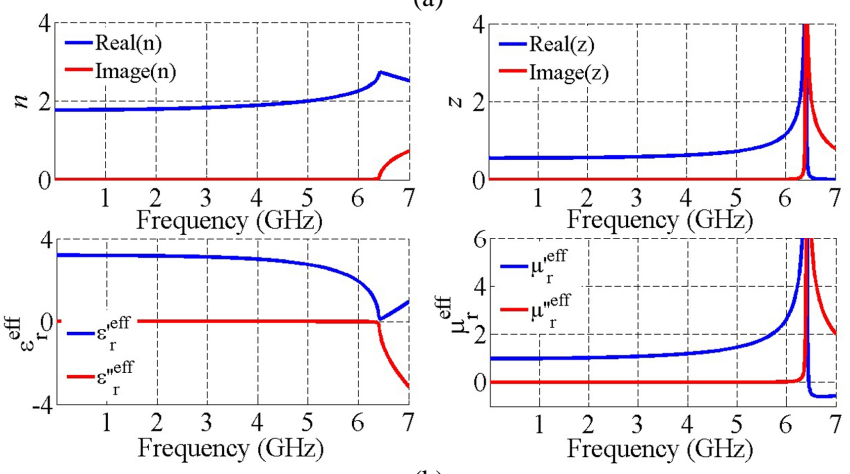

(b)

Fig. 5. (a) Magnitude and phase of the simulated $\mathrm{S}$ parameters for the unit cell in Fig. 4. (b) Retrieved index, impedance, permittivity and permeability.

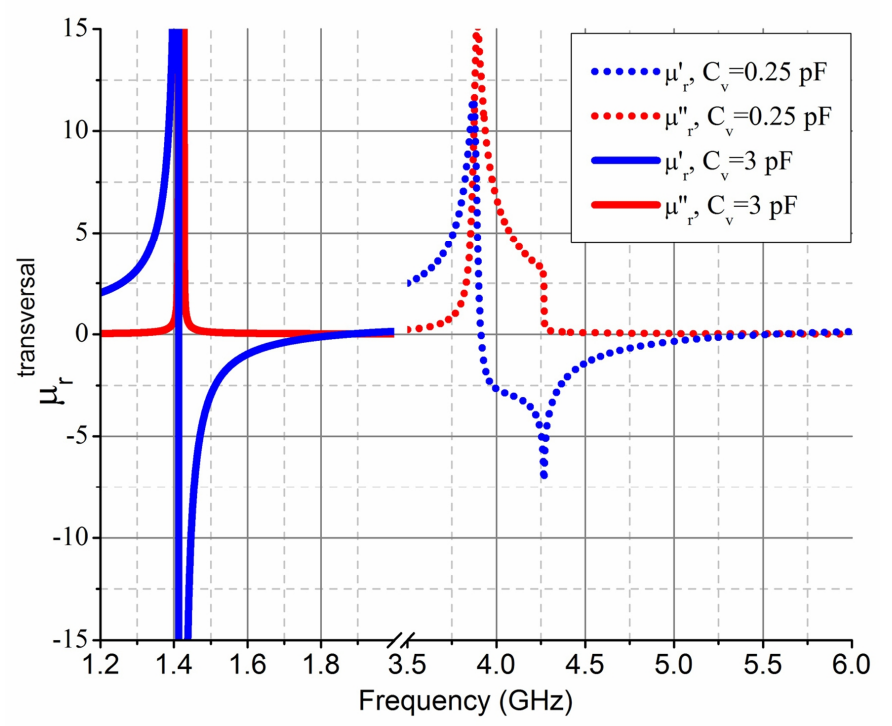

Fig. 6. Effect of different capacitance loadings $(\mathrm{Cv})$ on the resonance behavior of the transversal permeability.

\section{Varactor-loaded ESRR (VLESRR)}

It is clear that an SRR or ESRR, as a metamaterial resonator, can be simply modeled by an LC tank circuit [10], [17]. In fact, the strip behaves as an inductance $\left(L_{E S R R}\right)$ while the gap (split) acts as a capacitance $\left(C_{E S R R}\right)$. Nevertheless, the gap capacitance $\left(C_{E S R R}\right)$ has often small values and the associated magnetic moment is very weak. An inspection over the single layer ESRR-loaded SIW of Fig. 2 reveals that the gap of the proposed ESRR is available for external loadings. In this regard, the effect of $C_{E S R R}$ on the frequency response of the unit cell in Fig. 4 can be investigated by loading the gap with an external variable capacitor $\left(C_{v}\right)$. The corresponding effective constitutive parameters for two different capacitance loadings are obtained and shown in Fig. 6. As can be seen, the permeability is negative from about 3.9 to $5.5 \mathrm{GHz}$ for $C_{v}=0.25 \mathrm{pF}$ while for $C_{v}=3 \mathrm{pF}$, this range is 1.4 to $1.9 \mathrm{GHz}$. In fact, higher capacitance values increase the displacement loop current in the ESRR, resulting in a stronger magnetic moment. To better illustrate the permeability characteristics of the proposed resonator, the ESSR unit cell is separately simulated for three different capacitance loadings and the complex permeability constant is explored for each case, Fig. 7. The curves in Fig. 7 are represented in normalized frequency $\left(f / f_{0}\right)$ mainly to provide a clear comparison of the results. As can be seen, the bandwidth of negative effective

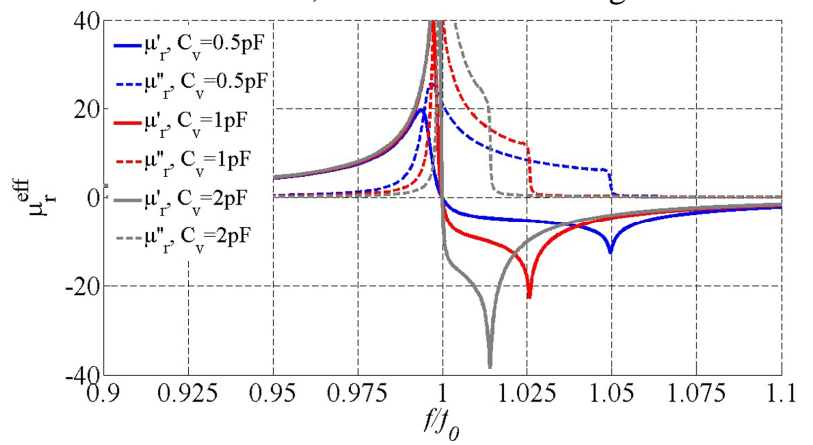

Fig. 7. Effective transversal permeability as a function of the normalized frequency $\left(f / f_{0}\right)$ for $C_{v}=0.5,1$ and $2 p F$. 
transversal permeability is decreased for increasing capacitance values, which is a direct consequence of the increased quality factor of resonators $\left(\mathrm{Q}_{\mathrm{r}}\right)$.

The above discussion that explained a capacitor-loaded ESRR is a key strategy to realize a tunable band of negative permeability. Frequency agility of the proposed ESRR for different capacitance values is shown in Fig. 8. The bandwidth of negative permeability in $\mathrm{GHz}$ for each $C_{v}$ is separately depicted on the associated bar.

A suitable element to provide a variable capacitor is the varactor diode, which works through a reverse bias voltage applied to it. Concerning the ESRR topology, a bias configuration is designed to load the varactor diodes, Fig. 9. A DC bias capacitor $\left(C_{\text {bias }}\right)$ is required to avoid short circuiting.

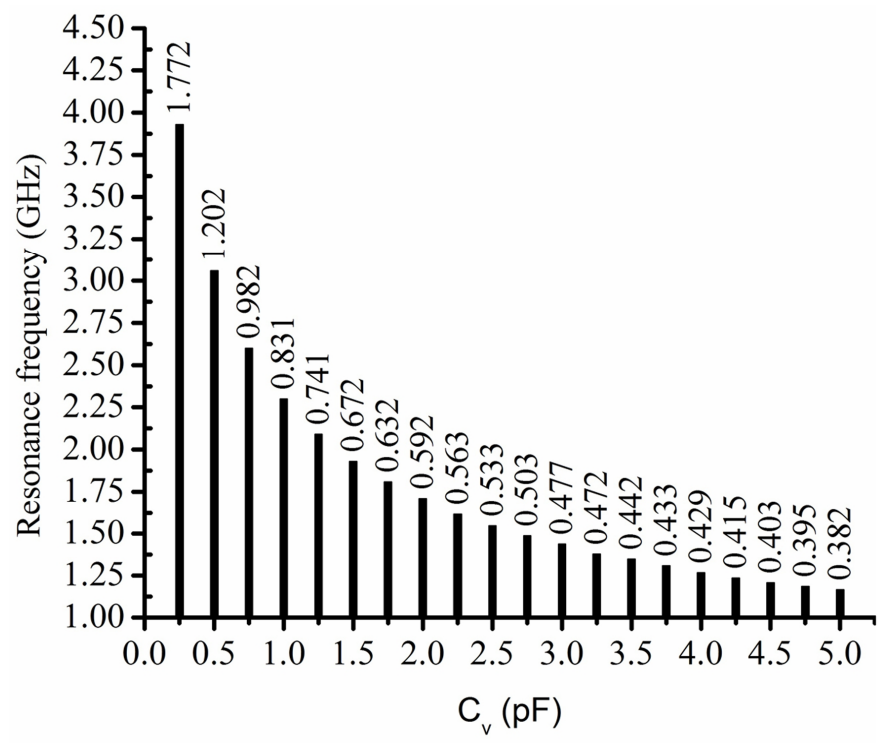

Fig. 8. Frequency agility of the ESRR for different $C_{v}$ s. The numbers above each bar represents the associated bandwidth of negative permeability in $\mathrm{GHz}$.

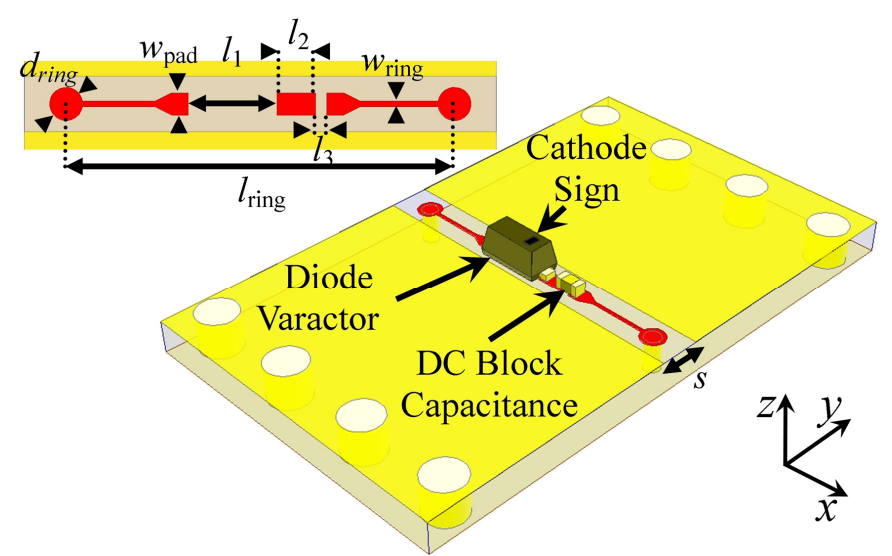

Fig. 9. Schematic of the proposed VLESRR-based SIW unit cell. The varactor scheme follows SC-79 package dimensions from Skyworks Corp. with a bias capacitor of $C_{\text {bias }}=22 p F$. the parameters are: $l_{\text {ring }}=7 \mathrm{~mm}, w_{\text {ring }}=0.1 \mathrm{~mm}, l_{1}=$ $1.6 \mathrm{~mm}, l_{2}=0.7 \mathrm{~mm}, l_{3}=0.2 \mathrm{~mm}, w_{\text {pad }}=0.4 \mathrm{~mm}, s=1 \mathrm{~mm}$ and $d_{\text {ring }}=0.4 \mathrm{~mm}$.

\section{SIW LOADED BY VLESRRS}

\section{A. Theoretical Analysis}

To analyze wave propagation in an SIW in presence of ESRRs, two sets of studies should be considered. In the first step, the effect of ESRRs on the circumference media should be considered (already discussed in section II). We found that the ESRRs could make a medium with an effective uniaxial permeability and an approximate isotropic permittivity. Next, wave mechanism in an SIW filled by a dielectric substrate with effective constitutive parameters should be investigated. To begin the analysis, the two rows of vias in an SIW can be replaced by two perfect electric walls transforming the SIW to an RWG with the same height and an equivalent width, [12]. Now, the problem of wave propagation in an RWG filled by an anisotropic material with effective constitutive parameters becomes our goal. The electric and magnetic field distribution inside the waveguide must satisfy source-free Maxwell's equations,

$$
\begin{aligned}
& \nabla \times \vec{E}=-j \omega \bar{\mu} \vec{H} \\
& \nabla \times \vec{H}=j \omega \bar{\varepsilon} \vec{E} \\
& \bar{\mu}=\mu_{0} \bar{\mu}_{r}=\mu_{0}\left[\begin{array}{ccc}
\mu_{x x} & 0 & 0 \\
0 & \mu_{y y} & 0 \\
0 & 0 & \mu_{z z}
\end{array}\right] \\
& \bar{\varepsilon}=\varepsilon_{0} \bar{\varepsilon}_{r}=\varepsilon_{0}\left[\begin{array}{ccc}
\varepsilon_{x x} & 0 & 0 \\
0 & \varepsilon_{y y} & 0 \\
0 & 0 & \varepsilon_{z z}
\end{array}\right]
\end{aligned}
$$

Where $\bar{\mu}$ and $\bar{\varepsilon}$ are general tri-axial permeability and permittivity tensors of the medium, respectively. By substituting (8) into (9) we have,

$$
\nabla \times\left\{\frac{1}{-j \omega} \bar{\mu}^{-1} \nabla \times \vec{E}\right\}=j \omega \vec{\varepsilon} \vec{E}
$$

In the dominate $\mathrm{TE}_{10}$ mode and for the propagation in the $+x$ direction, see the coordinates in Fig. 3, we have

$$
\begin{aligned}
& \vec{E}=\hat{a}_{z} E_{z}(y) e^{-j k_{x} x} \\
& \vec{H}=\hat{a}_{x} H_{x}(y) e^{-j k_{x} x}+\hat{a}_{y} H_{y}(y) e^{-j k_{x} x}
\end{aligned}
$$

Using the electric field expression of (13) and the permeability tensor of (10) in (12) leads to

$$
\nabla \times\left\{\left[\begin{array}{ccc}
\mu_{x x}^{-1} & 0 & 0 \\
0 & \mu_{y y}^{-1} & 0 \\
0 & 0 & \mu_{z z}^{-1}
\end{array}\right] \times\left[\begin{array}{c}
\partial E_{z}(y) e^{-j k_{x} x} / \partial y \\
-\partial E_{z}(y) e^{-j k_{x} x} / \partial x \\
0
\end{array}\right]\right\}=k_{0}^{2} \bar{\varepsilon}_{r} \vec{E}
$$


Where $k_{0}^{2}=\omega^{2} \mu_{0} \varepsilon_{0}$ is the free space wave number. The above equation can be simply solved to determine $E_{z}(y)$ and the complex propagation factor, $k_{x}$,

$$
\begin{aligned}
& \hat{a}_{x}\left(\mu_{y y}^{-1} \frac{\partial^{2} E_{z}(y) e^{-j k_{x} x}}{\partial x \partial z}\right)+\hat{a}_{y}\left(\mu_{x x}^{-1} \frac{\partial^{2} E_{z}(y) e^{-j k_{x} x}}{\partial y \partial z}\right) \\
& -\hat{a}_{z}\left(\mu_{y y}^{-1} \frac{\partial^{2} E_{z}(y) e^{-j k_{x} x}}{\partial x^{2}}+\mu_{x x}^{-1} \frac{\partial^{2} E_{z}(y) e^{-j k_{x} x}}{\partial y^{2}}\right) \\
& =\hat{a}_{z} k_{0}^{2} \varepsilon_{z z} E_{z}(y) e^{-j k_{x} x}
\end{aligned}
$$

Since $E_{z}$ is not a function of $z$, the first two terms in (15) will be eliminated. Equating the components in the $z$ direction results in

$$
\frac{\partial^{2} E_{z}(y) e^{-j k_{x} x}}{\partial y^{2}}=\left(\frac{\mu_{x x}}{\mu_{y y}} k_{x}^{2}-\mu_{x x} \varepsilon_{z z} k_{0}^{2}\right) E_{z}(y) e^{-j k_{x} x}
$$

Concerning the corresponding boundary conditions (the tangential electric field is zero at PEC walls), the above differential equation can be simply solved to

$$
E_{z}(y)=A \sin \left(k_{y} y\right) e^{-j k_{x} x}, \quad k_{y}=\frac{1 \times \pi}{w_{\text {equ }}}
$$

Where

$$
\begin{aligned}
& k_{y}^{2}=\mu_{x x} \varepsilon_{z z} k_{0}^{2}-\frac{\mu_{x x}}{\mu_{y y}} k_{x}^{2} \\
& k_{x}= \pm \sqrt{\mu_{y y} \varepsilon_{z z}\left(k_{0}^{2}-\frac{\pi^{2}}{\varepsilon_{z z} \mu_{x x} w_{e q u}^{2}}\right)}
\end{aligned}
$$

Here, $k_{x}$ is the complex propagation factor and depends on the transversal and longitudinal permeability constants $\left(\mu_{y y}\right.$ and $\mu_{x x}$, respectively) and the permittivity constant in the $z$ direction $\left(\varepsilon_{z z}\right)$. The choice of a solution with the positive or negative sign in (19) should be physically meaningful; i.e. the resulting waves should attenuate as they propagate along the guide. Generally, the two possible solutions for $k_{x}$ are as follows

$$
\begin{aligned}
& k_{x}^{2}=\left|k_{x}^{2}\right| e^{j \Phi}, \quad \Phi \in(-\pi, \pi) . \\
& k_{x 1}=\sqrt{\left|k_{x}^{2}\right|} e^{j \frac{1}{2} \Phi}=\beta_{x 1}-j \alpha_{x 1} \\
& k_{x 2}=\sqrt{\left|k_{x}^{2}\right|} e^{j\left(\frac{1}{2} \Phi+\pi\right)}=\beta_{x 2}-j \alpha_{x 2}
\end{aligned}
$$

Where $\beta_{x 1}, \beta_{x 2}$ and $\alpha_{x 1}, \alpha_{x 2}$ are the propagation and attenuation constants and will always have opposite sings. Nevertheless, the solution representing the attenuated waves should be first chosen; i.e. $\alpha_{x}>0$. This is later used to obtain the proper solution for $\beta_{x}$ and consequently $k_{x}$. In this case, losses are first considered with complex quantities: $\mu_{y y}=\mu_{y y}^{\prime}$ - ju ${ }^{\prime \prime}{ }_{y y}, \mu_{x x}=\mu_{x x}^{\prime}-j \mu^{\prime \prime}{ }_{x x}$ and $\varepsilon_{z z}=\varepsilon_{z z}^{\prime}-j \varepsilon_{z z}^{\prime \prime}$. Regarding the complex quantities and the complex propagation factor as $k_{x}=\beta_{x}-j \alpha_{x}$, the square of the propagation factor can be rewritten as

$$
\begin{aligned}
& k_{x}^{2}=R+j I \\
& \Phi=\tan ^{-1}\left(\frac{I}{R}\right)
\end{aligned}
$$

\section{Where}

$$
\begin{aligned}
& R=A E-B F \\
& I=-B E-A F \\
& A=\mu_{y y}^{\prime} \varepsilon_{z z}^{\prime}-\mu_{y y}^{\prime \prime} \varepsilon_{z z}^{\prime \prime} \\
& \mathrm{B}=\mu_{y y}^{\prime} \varepsilon_{z z}^{\prime \prime}+\mu_{y y}^{\prime \prime} \varepsilon_{z z}^{\prime} \\
& C=\mu_{x x}^{\prime} \varepsilon_{z z}^{\prime}-\mu_{x x}^{\prime \prime} \varepsilon_{z z}^{\prime \prime} \\
& D=\mu_{x x}^{\prime} \varepsilon_{z z}^{\prime \prime}+\mu_{x x}^{\prime \prime} \varepsilon_{z z}^{\prime} \\
& E=k_{0}^{2}-\frac{C\left(\pi / w_{e q u}\right)^{2}}{C^{2}+D^{2}} \\
& F=\frac{D\left(\pi / w_{e q u}\right)^{2}}{C^{2}+D^{2}}
\end{aligned}
$$

In the lossless case, the phase (propagation) constant, $\beta_{x}$, is simplified to

$$
\begin{aligned}
& \beta_{x}= \pm k_{0} \sqrt{\mu_{y y}^{\prime} \varepsilon_{z z}^{\prime}\left(1-\left(\frac{f_{c}}{f}\right)^{2}\right)} \\
& f_{c}=\frac{\pi}{2 w_{\text {equ }} \sqrt{\mu_{x x}^{\prime} \varepsilon_{z z}^{\prime}}}
\end{aligned}
$$

The above expression is almost similar to the familiar form of wave propagation formula in a conventional RWG. For a regular material with positive parameters $\left(\mu_{y y}>0, \mu_{x x}>0\right.$ and $\varepsilon_{z z}>0$ ) and for $f>f_{c}, f=f_{c}$ and $f<f_{c}$, Eq. (22) illustrates the well-known highpass behavior in the waveguide.

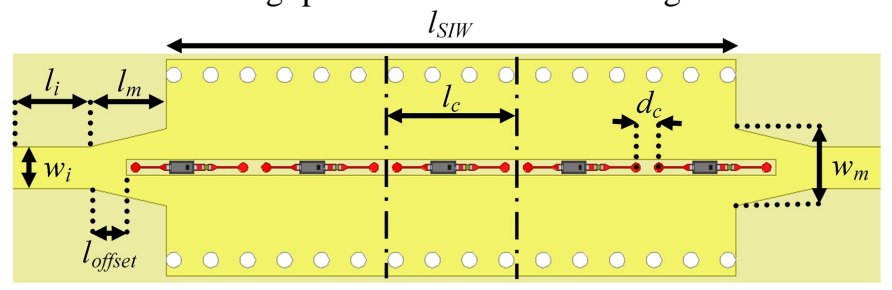

Fig. 10. An SIW structure loaded by five VLESRRs with the bias configuration of Fig. 9. The geometrical parameters are: $w_{\text {sIw }}=12 \mathrm{~mm}, \mathrm{w}_{\mathrm{m}}=5 \mathrm{~mm}, 1_{\mathrm{m}}=5$ $\mathrm{mm}, \mathrm{w}_{\mathrm{i}}=1.8 \mathrm{~mm}, \mathrm{l}_{\mathrm{i}}=5 \mathrm{~mm}, l_{c}=8.5 \mathrm{~mm}, d_{c}=1.5 \mathrm{~mm}$ and $l_{\text {offset }}=3 \mathrm{~mm}$. 


\section{B. Propagation below the Cutoff Frequency $\left(f_{c}\right)$}

In this section, wave mechanism in a VLESRR- based SIW is investigated in more details. Toward this end, an SIW structure loaded by five VLESRRs with the bias configuration of Fig. 9 is sketched in Fig. 10. The structure is implemented on a substrate of Rogers $\mathrm{RO} 4003$ with a relative permittivity $\varepsilon_{r}=3.55$ and a thickness of 32 mil. The vias of the SIW have a diameter of $d=1 \mathrm{~mm}$ and a center-to-center spacing of $p=$ $2.4 \mathrm{~mm}$; resulting in $f_{c} \approx 7 \mathrm{GHz}$. A microstrip-to- SIW transition is also designed for the best possible matching.

For a better illustration, wave propagation is first discussed in the lossless case where the use of Eq. (24) provides an engineered insight on the wave mechanism of an SIW filled by a uniaxial effective material. The constitutive parameters obtained from a VLESRR unit cell can be generalized to achieve a homogeneous uniaxial medium. Finally, the propagation constant $\left(k_{x}\right.$ or $\beta_{x}$ ) of an SIW filled by such a medium can be simply calculated by substituting the extracted constitutive parameters in Eq. (24). Based on this equation, if the transversal permeability $\left(\mu_{y y}\right)$ is negative below $f_{c}$ (while the remaining components of the constitutive parameters are positive), a passband will be established. The constitutive parameters of Fig. 11 correspond to a VLESRR unit cell with $C_{v}=0.5 \mathrm{pF}$. It can be seen that the transversal permeability $\left(\mu_{y y}\right)$ has a resonance frequency at $3.06 \mathrm{GHz}$ and is negative in a frequency band from 3.06 to $4.26 \mathrm{GHz}$. In addition, the effective permittivity $\left(\varepsilon_{z z}\right)$ resonates at about $3.18 \mathrm{GHz}$, but remains positive in the whole frequency band of interest. Now, based on Eq. (24) and the constitutive parameters of Fig. 11 (a), a propagation band is expected from 3.06 to 4.26 $\mathrm{GHz}(1.2 \mathrm{GHz}$ bandwidth); i.e. at the frequency interval where $\mu_{y y}<0$, Fig. 11 .

To illustrate the propagation constant in the lossless case, Eq. (24) must be explored with the correct sign. If we assume propagation in the $+x$ direction, $\alpha_{x}$ should always be positive to realize evanescent waves. Fig. 12 (a) shows the propagation constant of Eq. (24) with a positive sign. As can be seen, for $f<5.4 \mathrm{GHz}, \alpha_{x}$ is negative and thus a negative sign should be adopted in Eq. (24) to result the correct solution in this frequency range, Fig. 12 (b).

In this case, the phase constant $\left(\beta_{x}\right)$ is real and the attenuation constant $\left(\alpha_{x}\right)$ is zero, Fig. 12. On the contrary, there is no propagation below $3.06 \mathrm{GHz}$ and from $4.26 \mathrm{GHz}$ to $6.3 \mathrm{GHz}$, where the phase constant is also zero.

In addition, propagation will also begin above $6.3 \mathrm{GHz}$, which means that the cutoff frequency $\left(f_{c}\right)$ of an SIW loaded by a single VLESRR is $6.3 \mathrm{GHz}$; slightly lower than the initial unloaded SIW ( $\approx 7 \mathrm{GHz}$ ). This is due to the relative permittivity of the structure at the cutoff which is effectively increased to about 4, Fig. 11 (a).

Figure 13 demonstrates the frequency response of an SIW loaded by a single VLESSR with $C_{v}=0.5 \mathrm{pF}$. As can be seen, a propagation band is achieved near $3.375 \mathrm{GHz}$ from 3.325 to $3.6 \mathrm{GHz}$ (a 3-dB bandwidth of $275 \mathrm{MHz}$ ). In addition, the result in Fig. 13 nicely correlates with the propagation constant of Fig. 12; though there are some differences in the
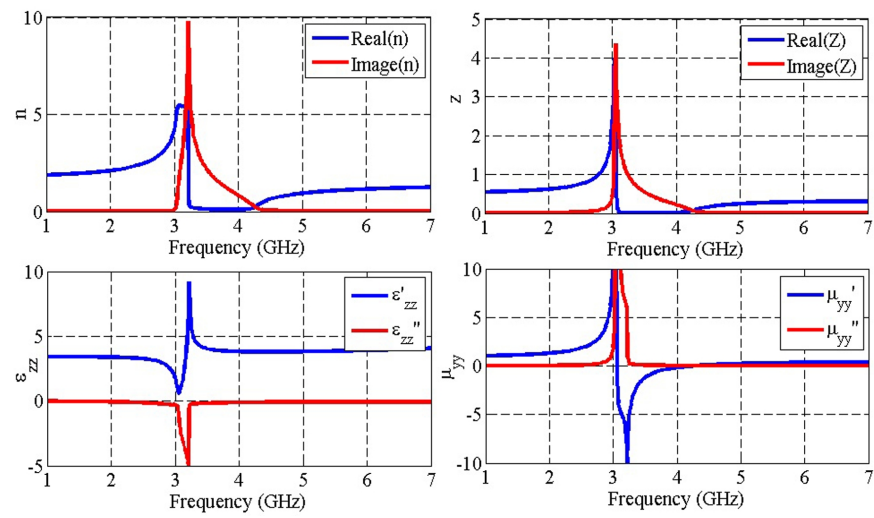

(a)

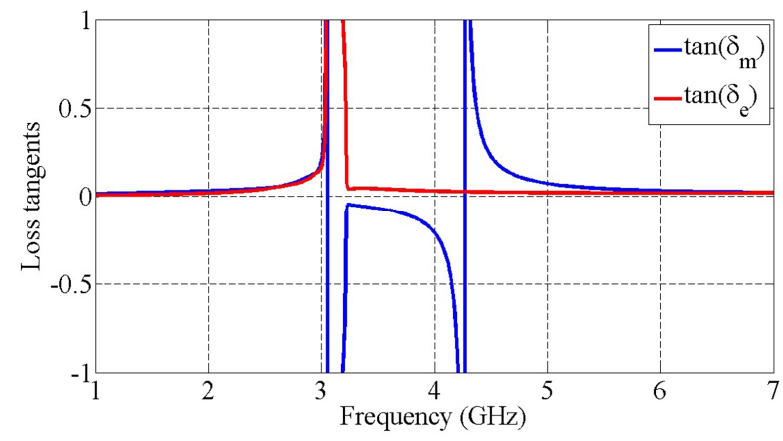

(b)

Fig. 11. Constitutive parameters of a VLESRR unit cell with $C_{v}=0.5 \mathrm{pF}$. (a) Transversal permeability and $z$-axis permittivity. (b) Electric and magnetic loss Tangent along $z$ and $y$ axes respectively.

passband bandwidth. In fact, the propagation constant (Fig. 12) shows a passband of $1.2 \mathrm{GHz}$ while the $\mathrm{S}$ - parameter results of Fig. 13 show a 3-dB bandwidth of $275 \mathrm{MHz}$. This is due to the introduced magnetic and electric losses of the ESRR. Here, the propagation constant in the presence of the losses will be explored. In this regard, Eq. (19) is applied which considers wave propagation constant $\left(k_{x}\right)$ in the general lossy case by substituting $\mu_{y y}=\mu_{y y}^{\prime}-j \mu^{\prime \prime}{ }_{y y}$ and $\varepsilon_{z z}=\varepsilon_{z z}^{\prime}-j \varepsilon_{z z}^{\prime \prime}$. For simplicity and without any loss of generality, $\mu_{x x}$ is assumed unity.

An analytical method like the one in [6] can be used to choose for Eqs. (21-a) or (21-b). Nevertheless, if $k_{x}$ is sketched during the frequency band of interest, we can obtain the correct solution based on these two equations. Similar to the discussion on the lossless case, Eq. (21-a) is adopted for $f$ $>5.4 \mathrm{GHz}$ while Eq. (21-b) should be used for $f<5.4 \mathrm{GHz}$.

In this case, phase and attenuation constants $\left(\beta_{x}\right.$ and $\left.\alpha_{x}\right)$ are sketched in Fig. 14 (a) with a more detailed sketch to provide a better insight, Fig. 14 (b). The propagation constant in the lossy case has the same behavior as the lossless case, though wave mechanism can be investigated more accurately. Specifically, $\alpha_{x}$ is not zero in the propagation band resulting in a reduced passband. The corresponding frequency behavior of Fig. 14 obtained from the extracted constitutive parameters and Eq. (19) well describes the frequency response of an SIW loaded by a single ESRR, Fig. 13. Thus, an SIW loaded by ESRR(s) can be well analyzed by the extracted constitutive parameters obtained from an ESRR unit cell. 


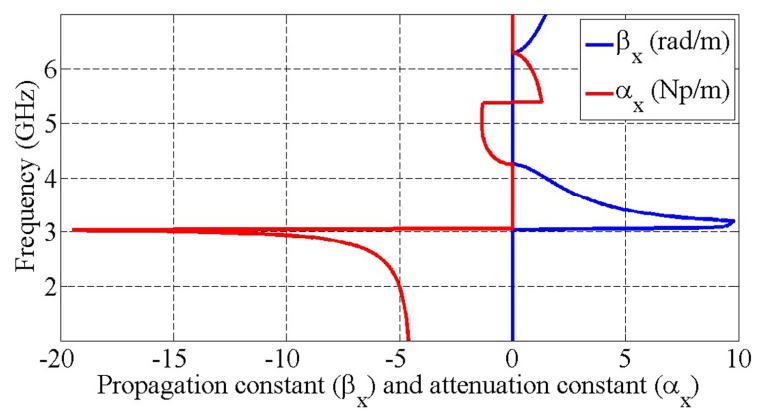

(a)

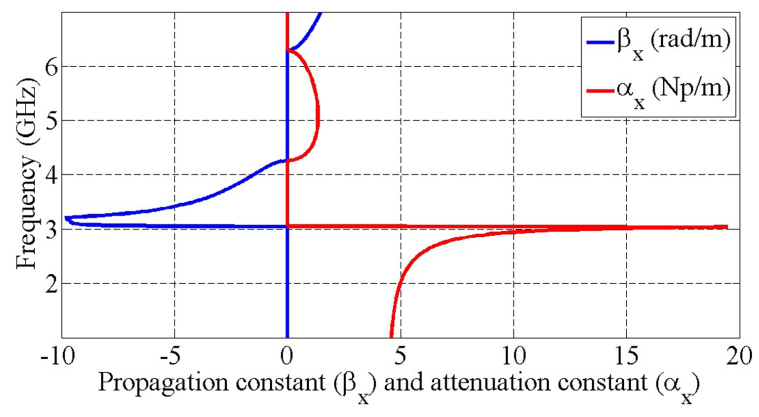

(b)

Fig. 12. Propagation constant and attenuation constant in loss-less case in the SIW loaded by material with characteristics shown in Fig. 11 (a).

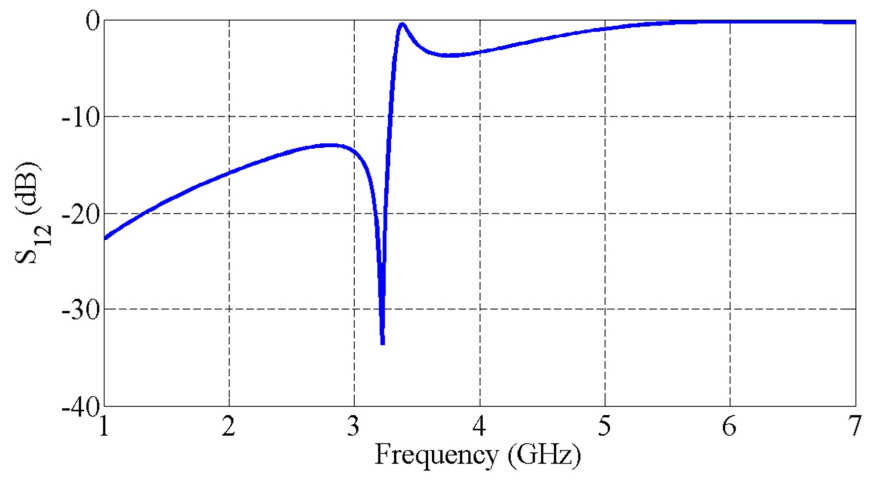

Fig. 13. Frequency response of the SIW of Fig. 10 with $C_{v}=0.5 \mathrm{pF}$.

In addition to the above analyses, a simple method to predict the frequency behavior of an ESRR based SIW transmission line from its unit cell is to make use of the corresponding parameters along with losses. For example, Fig. 11 demonstrates that for $f<f_{c}$ and when the transversal permeability is negative, $\beta_{x}$ will be real; this means that a passband can be formed in the interval where $\mu_{y y}<0$. But in Fig. 13 or 15 the corresponding passband is narrower compared to the interval where $\mu_{y y}$ is negative. In this case, the losses, including electric $\left(\delta_{e}\right)$ and magnetic $\left(\delta_{m}\right)$, play a key role in specifying the propagation band width. In Fig 11(b) the losses are small around $3.3 \mathrm{GHz}$. Below this frequency, both losses are increased sharply, and above that, the magnetic loss is only present though it gradually becomes higher. This implies a sharper rejection below the propagation band.

A more distinct passband can be achieved if more losses are introduced to the right edge of the passband in Fig. 13. Toward this end, the host SIW can be loaded by N numbers of ESRRs with $\mathrm{N}=2,3 \ldots$ The frequency response

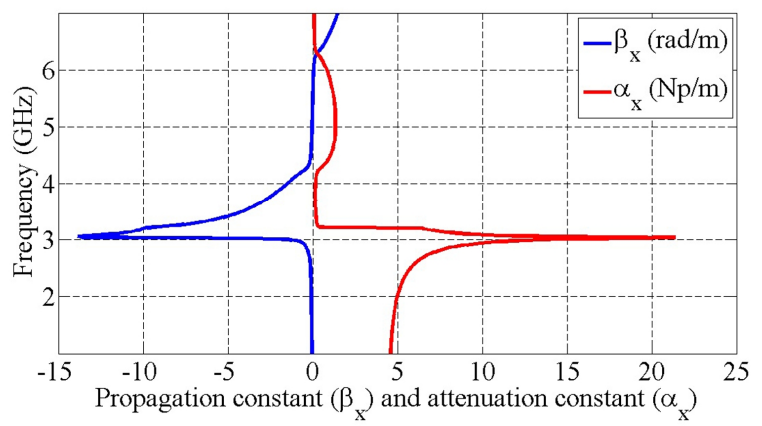

Fig. 14. Propagation constant and attenuation constant in lossy case for the SIW loaded by material with characteristics shown in Fig. 11.

for an SIW loaded by two to seven ESRRs is sketched in Fig. 15 where it can be seen that the loaded SIW maintains the bandpass-like (THROUGH) response; though the rejection of the responses becomes sharper at both edges as $\mathrm{N}$ increases. This is evident in view of the additional attenuation of each ESRR element which is added to the overall frequency response.

As can be seen from Fig. 14 and for a single arbitrary point in the passband region below $f_{c}$, the slope of the line which passes through the origin and that point $\left(v_{p}=\beta_{x} / \omega\right)$ on the $\beta_{x}$ curve is antiparallel to the line tangential to $\beta_{x}$ in that point $\left(v_{g}=d \omega / d \beta_{x}\right)$. This means that the propagation of waves is in the form of backward waves, [18].

Furthermore a simple method to prove the existence of backward-wave propagation is to measure the phase $S_{21}$ of between two different physical lengths of the loaded SIW ( $L_{1}$ and $L_{2}$, where $L_{1}>L_{2}$ ). In backward-wave passband $\left(\varphi_{S_{21}}\left(L_{2}\right)-\varphi_{S_{21}}\left(L_{1}\right)>0\right),[6,10]$. This is shown in Fig. 16 for two different physical lengths of the loaded SIW in backward-wave propagation region.

The above discussion explained the effect of an SIW loaded by VLESRR(s) for $C_{v}=0.5 p F$ where $C_{v}$ is the capacitor value of the associated varactor diode(s). Nevertheless, it should be noted that the structure can be modified to represent tunable passbands if $C_{v}$ is changed. In this case, a variable reverse bias voltage can be applied to the varactors, which results in different $C_{v}$ s and consequently different regions of negative transversal permeability, Figs. 7 and 8. Thus, the passbands below $f_{c}$ can be continuously tuned due to a continuous variable voltage. Here, SMV2019-079LF varactor diodes from Skyworks Corporation are used, which completely covers our

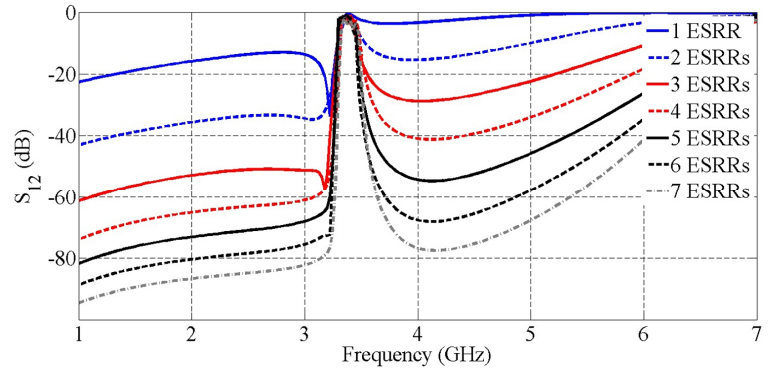

Fig. 15. Return losses of the SIW with 1 to 7 VLESRRs. 


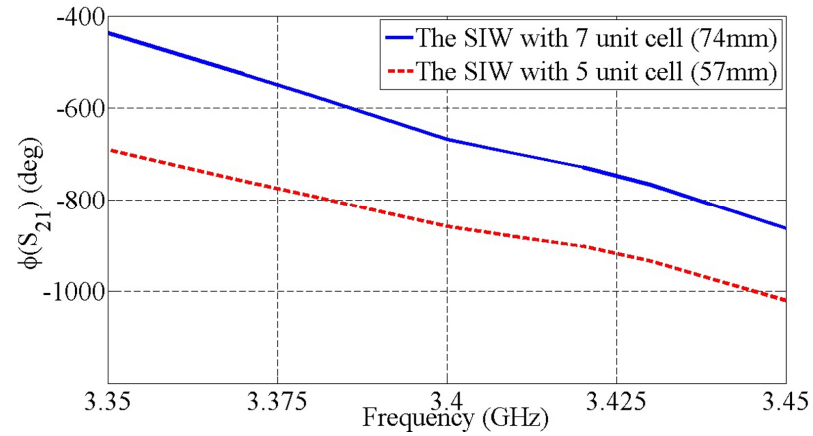

Fig. 16. Phase difference comparison to prove existence of back-ward wave propagation for the structure.

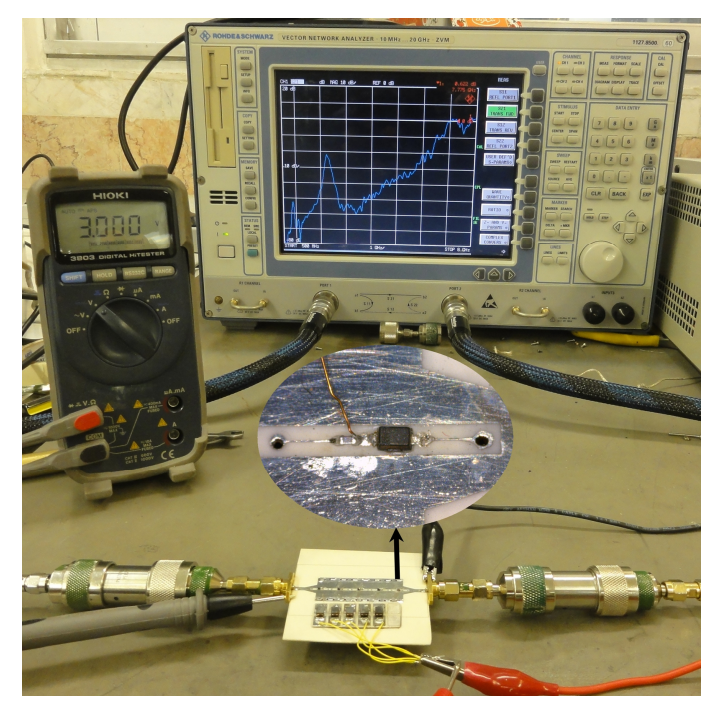

(a)

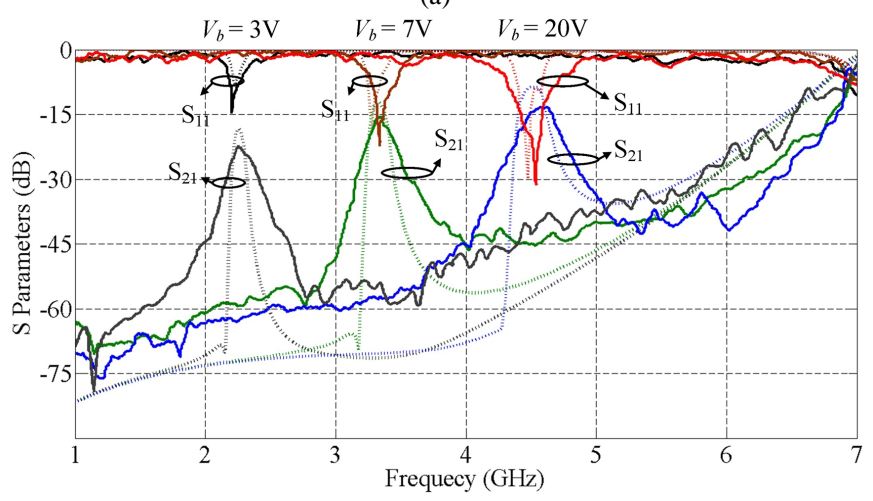

(b)

Fig. 17. (a) Experimental setup for the VLESRR loaded SIW. (b) Measured (solid lines) and simulated (dotted lines) frequency responses of the circuit for three sample voltages of 3, 7 and $20 \mathrm{~V}$.

capacitance range of interest, though they suffer from a bit higher series resistor $\left(r_{s}\right)$.

Figure 17 (a) shows the fabricated prototype of an SIW loaded by five VLESSRs under the experimental setup and for a revers bias voltage of $3 \mathrm{~V}$. The corresponding measured frequency response of the circuit is also shown and compared with the simulated results for three sample bias voltages, Fig. 17 (b). It should be pointed out that the simulations are performed to account for the series resistor $\left(r_{s}=1 \Omega\right)$. As seen, the two results are in good agreement. Obviously, the losses introduced in the passband can be drastically reduced if lower loss varactor diodes are used.

\section{Propagation above the Cutoff Frequency $\left(f_{c}\right)$}

The effect of negative transversal permeability below the cutoff frequency $\left(f_{c}\right)$ of an SIW loaded by VLESRRs was discussed in the previous section. Here, wave mechanism above $f_{c}$ is investigated. In this regard, an SIW is designed and loaded by the VLESRRs whose resonance frequency is set above $f_{c}$ of a host SIW. Both SIW and VLESRR structures are quite similar to former designs except for a substrate of Rogers RO6010 with a relative permittivity $\varepsilon_{r}=10.2$ and a thickness of 50 mil. The width of the SIW is also increased to $w_{S I W}=25 \mathrm{~mm}$. In addition, the vias of the SIW have a diameter of $d=0.4 \mathrm{~mm}$ and a center-to-center spacing of $p=$ $1.7 \mathrm{~mm}, l_{\text {offset }}=0.1 \mathrm{~mm}$ and the length of the SIW $l_{\text {SIW }}=32.8$ $\mathrm{mm}$; resulting in an unloaded SIW with $f_{c} \approx 1.9 \mathrm{GHz}$. A microstrip-to-SIW transition is also designed for the best possible matching $\left(w_{m}=8 \mathrm{~mm}, l_{m}=5 \mathrm{~mm}, w_{i}=2.5 \mathrm{~mm}, l_{i}=5\right.$ $\mathrm{mm})$. Based on Fig. 9, the unit cell parameters are: $l_{\text {ring }}=3.4$ $\mathrm{mm}, w_{\text {ring }}=0.1 \mathrm{~mm}, l_{1}=1.2 \mathrm{~mm}, l_{2}=0.8 \mathrm{~mm}, l_{3}=0.15 \mathrm{~mm}$ and $w_{\text {pad }}=0.4 \mathrm{~mm}$, with a bias capacitor of $C_{\text {bias }}=22 \mathrm{pF}$.

To investigate wave mechanism in this case, the effective constitutive parameters for a VLESRR with capacitance loading of $C_{v}=l p F$ are obtained, Fig. 18.

Based on Eq. (24), it is expected to have a stopband above $f_{c}$ of an SIW in the negative transversal permeability region; i.e. where $\mu_{y y}<0$. Here, the general lossy case of a negative effective uniaxial permeability medium above $f_{c}$ of an SIW is considered. Using Eq. (19) and the extracted effective constitutive parameters, phase and attenuation constants $\left(\beta_{x}\right.$ and $\alpha_{x}$ ) for the SIW loaded by a material whose characteristics are sketched in Fig. 18 are obtained in Fig. 19. A similar procedure as the one in the previous section is adopted to explain for the correct sign of the propagation constant in Eqs. (21-a) or (21-b). Here for $f>1.5 \mathrm{GHz}$, Eq. (21-a) will be applied while for $f<1.5 \mathrm{GHz}$, Eq. (21-b) will be the correct solution. The frequency response of the loadedSIW with five VLESRRs is explored. Figure 19 shows propagation band from about 1.4 to $3 \mathrm{GHz}$. The phase constant approaches zero and attenuation constant finds values from about 3 to $3.7 \mathrm{GHz}$ interpreted as a stopband. The propagation begins, again, above $3.7 \mathrm{GHz}$. The frequency response shown in Fig 20 also indicates a similar behavior. It is noted that the circuit can provide a continuous control over the frequency response and thus a fully electronic tunable notch transmission line is plausible. In this regard, the lower and upper $3 \mathrm{~dB}$ frequencies as well as the corresponding bandwidths for a number of bias voltages are summarized in Table 1.

So far the effect of negative transversal permeability above $f_{c}$ of the designed SIW has been explained. In the next step, now, we vary the bias voltage applied to the varactor diodes to tune the resulting stopband. Figure 21 illustrates the measured and simulated (with $r_{s}=1 \Omega$ ) frequency responses of the circuit for two sample bias voltages. 

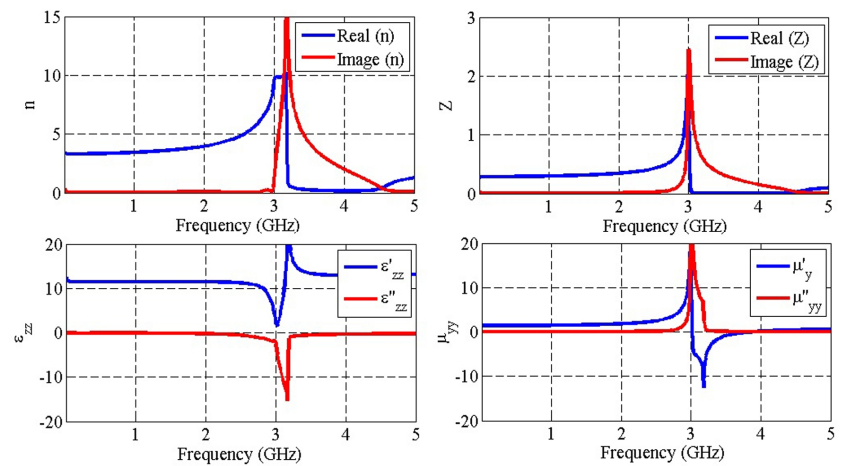

(a)

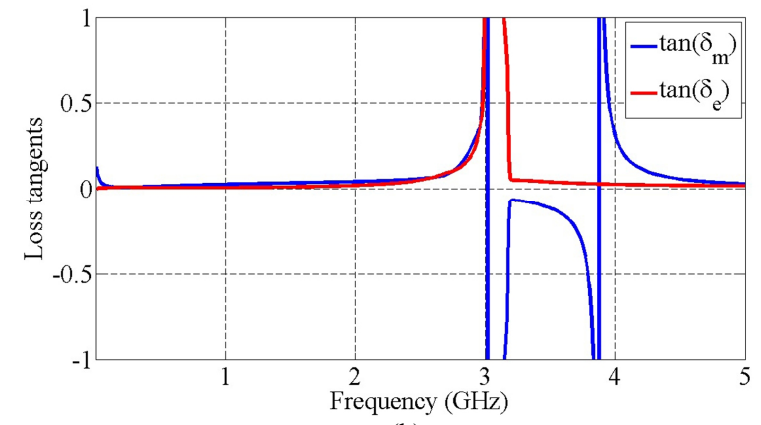

(b)

Fig. 18. Constitutive parameters of a VLESRR unit cell with $C_{v}=1 \mathrm{pF}$, with $\Delta y=3 \mathrm{~mm}, \Delta z=1.35 \mathrm{~mm}$ and $l_{c}=4.6 \mathrm{~mm}$. (a) Transversal permeability and $z$-axis permittivity. (b) Electric and magnetic loss Tangent along $z$ and $y$ axes respectively.

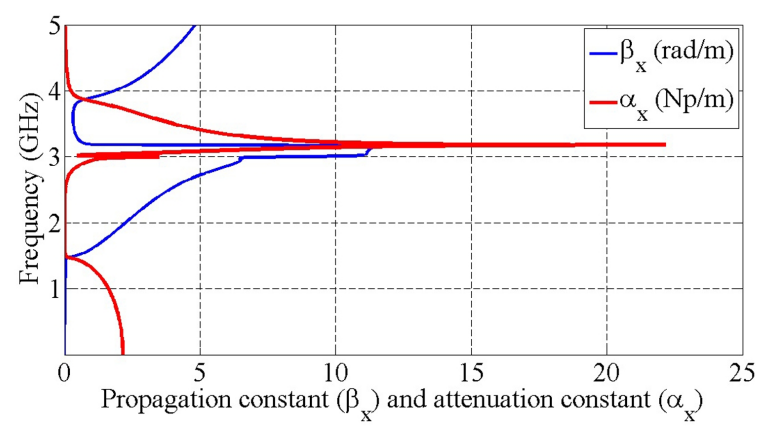

Fig. 19. Propagation constant and attenuation constant in lossy case for the SIW loaded by material with characteristics shown in Fig. 18.

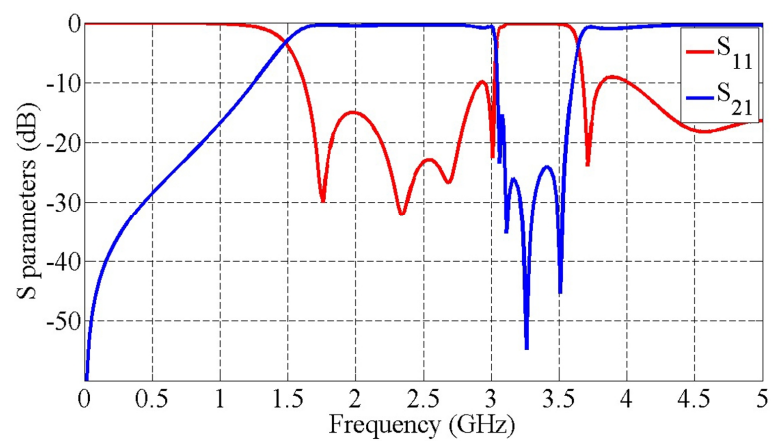

Fig. 20. Frequency response of the SIW with $C_{\nu}=1 \mathrm{pF}$.

\section{CONCLUSION}

A single layer embedded SRR was proposed for the first time in this paper. The frequency behavior of the element was explored. Wave propagation and mechanism of ESRR- based

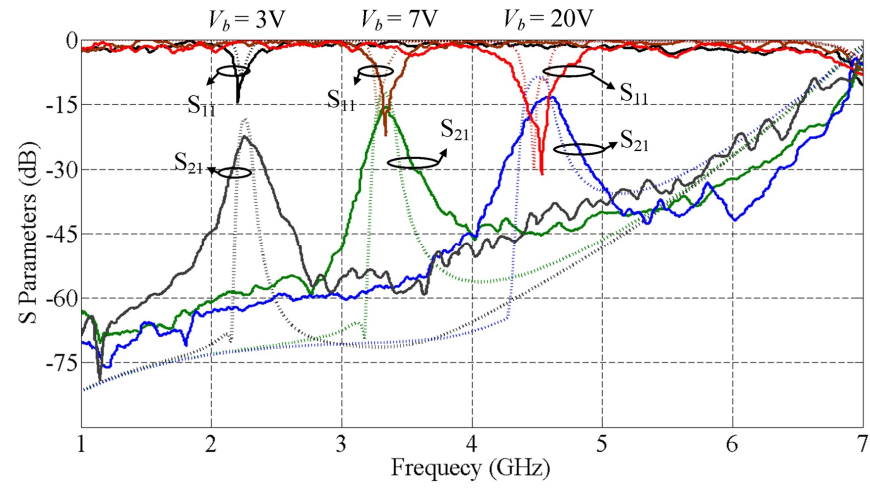

Fig. 21. Measured (solid lines) and simulated (dotted lines) frequency responses of the circuit for two sample voltages of 1 and 7 .

\begin{tabular}{cccc}
\hline \multicolumn{5}{c}{ Table 1. Frequency response of the VLESRR-loaded } \\
\multicolumn{4}{c}{ SIW TL for various reverse bias voltages $\boldsymbol{V}_{\boldsymbol{b}}(\mathbf{V})$} \\
\hline $\boldsymbol{V}_{\boldsymbol{b}}(\boldsymbol{V})$ & $\boldsymbol{C}_{\boldsymbol{v}}(\boldsymbol{p} \boldsymbol{F})$ & $\boldsymbol{f}_{\boldsymbol{c}-\text {-low- }} \boldsymbol{f}_{\boldsymbol{c} \text {-high }}(\mathbf{G H z})$ & $\boldsymbol{B W}(\mathbf{M H z})$ \\
$\mathbf{7}$ & 0.5 & $3.94-4.84$ & 900 \\
$\mathbf{4 . 3}$ & 0.75 & $3.34-4.12$ & 780 \\
$\mathbf{2 . 9}$ & 1 & $3.04-3.62$ & 580 \\
$\mathbf{1 . 7 5}$ & 1.25 & $2.79-3.31$ & 520 \\
$\mathbf{1}$ & 1.5 & $2.56-3.07$ & 510 \\
$\mathbf{0 . 5}$ & 1.75 & $2.39-2.90$ & 510 \\
$\mathbf{0 . 2}$ & 2 & $2.26-2.75$ & 490 \\
$\mathbf{0}$ & 2.22 & $2.15-2.63$ & 480 \\
\hline
\end{tabular}

SIW transmission lines was thoroughly discussed. It was also shown that the resonance behavior of the proposed ESRR can be controlled through the edge capacitance of the associated gap. Later, the ESRR was loaded by varactor diodes which could efficiently and continuously tune the resonance frequency. The resulting VLESRR was applied in SIWs. In this regard, two different cases were considered. First, five identical VLESRRs were used whose resonant frequencies were set below the cutoff frequency, $f_{c}$, of an unloaded SIW. This case led to a VLESRR- based SIW TL with tunable passbands below $f_{c}$. The next case discussed an SIW loaded by five VLESRRs whose resonance frequency was greater than the SIW $f_{c}$. This could be thought as the dual of the former case, somehow tunable stopbands are established above $f_{c}$. In the analytical studies for both cases, the propagation constant is obtained from a unit cell and then the substrate of the SIW is modeled by the resulting effective medium. This assumption is validated since the two results are quite compatible.

The electronic tunability provided in both circuits can realize selective frequency bands fast and accurately. Generally, the proposed transmission lines can be integrated with planar boards. The design procedure for both SIW and VLESRR structures is straightforward to select or eliminate a desired frequency band. Besides, low loss varactors may improve the performance of the proposed structures. Finally, fabricated prototypes for the two circuits with the available varactors were measured to validate the simulated and analytical results. 


\section{REFERENCES}

[1] C. A. Balanis, Advanced Engineering Electromagnetics. New York: Wiley, 1989.

[2] Li Yan, Wei Hong, Guang Hua, Jixin Chen, Ke Wu, and Tie Jun Cui, "Simulation and Experiment on SIW Slot Array Antennas," IEEE Microwave and Wireless Components Letters, Vol. 14, No. 9, pp. 446448, Sept. 2004.

[3] W. D'Orazio and Ke Wu, "Substrate-Integrated-Waveguide Circulators Suitable for Millimeter-Wave Integration," IEEE Trans. on Microwave Theory and Techniques, Vol. MTT 54, No. 10, pp. 3675-3680, Oct. 2006.

[4] Yuan Dan Dong; Tao Yang; Itoh, T.; , "Substrate Integrated Waveguide Loaded by Complementary Split-Ring Resonators and Its Applications to Miniaturized Waveguide Filters," Microwave Theory and Techniques, IEEE Transactions on , vol.57, no.9, pp.2211-2223, Sept. 2009.

[5] R. Marques, J. Martel, F. Mesa, and F. Medina, "Left-handed-media simulation and transmission of EM waves in subwavelength split-ringresonator-loaded metallic waveguides," Phys. Rev. Lett., pp. 183 901-183 904, Oct. 2002.

[6] Hrabar, S.; Bartolic, J.; Sipus, Z.; , "Waveguide miniaturization using uniaxial negative permeability metamaterial", Antennas and Propagation, IEEE Transactions on , vol.53, no.1, pp. 110- 119, Jan. 2005.

[7] Yuan Dan Dong; Tao Yang; Itoh, T.; , " Substrate Integrated Waveguide Loaded by Complementary Split-Ring Resonators for Miniaturized Diplexer Design," IEEE Microwave and Wireless Components Letters, Vol. 21, No. 1, pp. 10-12, Jan. 2011.

[8] Q. Cheng, and T.-J. Cui, "Realization of Left-Handed Transmission Structures Using the Substrate Integrated Waveguide Technology," in Proceedings of International Symposium on Biophotonics, Nanophotonics and Metamaterials, pp. 447-450, Hangzhou, China, Oct. 2006.

[9] Glubokov, A.; Budimir, D.; , "Compact multilayer SRR-loaded integrated waveguide filters on liquid crystal polymer substrate," Antennas and Propagation Society International Symposium, 2008. AP-S 2008. IEEE, vol., no., pp.1-4, 5-11 July 2008.

[10] Farzami, F.; Forooraghi, K.; Norooziarab, M.; , "Design and Modeling of a Miniaturized Substrate Integrated Waveguide Using Embedded SRRs," Antennas and Wireless Propagation Letters, IEEE, vol.10, no., pp.713716, 2011.

[11] Y. Cassivi, L. Perregrini, P. Arcioni, M. Bressan, K. Wu, \& G. Conciauro; "Dispersion characteristics of substrate integrated rectangular waveguide," IEEE Microw. Wireless Compon. Lett., vol. 12, no. 9, pp. 333-335, Sep. 2002.

[12] Deslandes, D.; Ke Wu, "Accurate modeling, wave mechanisms, and design considerations of a substrate integrated waveguide", MTT, IEEE Transactions on, vol.54, no.6, pp.2516-2526, June 2006.

[13] Farzami, F.; Forooraghi, K.; Norooziarab, M., "Miniaturization of a Microstrip Antenna Using a Compact and Thin Magneto-Dielectric Substrate," Antennas and Wireless Propagation Letters, IEEE, vol.10, no., pp. $1540,1542,2011$

[14] S. Hrabar and J. Bartolic, "Capacitively loaded loop as basic element of negative permeability meta material," in Proc. 2002 European Microwave, Milan, Italy, 2002, pp. 357-361.

[15] J. B. Pendry, A. J. Holden, D. J. Robbins, and W. J. Stewart. "Magnetism from conductors and enhanced nonlinear phenomena," IEEE Trans. Micr. Theory. Tech., vol. 47, no. 11, pp. 2075-1084, Nov. 1999.

[16] D. Smith, D. Vier, T. Koschny, and C. Soukoulis, "Electromagnetic parameter retrieval from inhomogeneous metamaterials," Physical Review E, vol. 71, p. 36617, 2005.

[17] Baena, J.D.; Bonache, J.; Martin, F.; Sillero, R.M.; Falcone, F.; Lopetegi, T.; Laso, M.A.G.; Garcia-Garcia, J.; Gil, I.; Portillo, M.F.; Sorolla, M.; , "Equivalent-circuit models for split-ring resonators and complementary split-ring resonators coupled to planar transmission lines," Microwave Theory and Techniques, IEEE Transactions on , vol.53, no.4, pp. 14511461, April 2005.

[18] C. Caloz and T, Itoh, "Electromagnetic Metamaterials: Transmission Line Theory and microwave Applications". New York, John Wiley \& Sons, 2005. 